\title{
Analysis of coupled bunch instability spectra
}

\author{
E. Shaposhnikova
}

\begin{abstract}
Beam spectra observed during the development of a coupled bunch instability contain information about the coupled bunch mode $n$, which describes the phase shift between adjacent bunch oscillations. This number indicates the possible frequency of the guilty impedance with accuracy up to an integer multiple of the bunch spacing frequency. However when there are many possible candidates with imprecisely known frequencies this can be insufficient. In this paper we discuss what additional information about the frequency of the source of instability can be obtained from the analysis of the unstable beam spectra envelope. This is applied to the measurements in the CERN SPS.
\end{abstract}

Presented at the Workshop on Instabilities of High intensity Hadron Beams in Rings, BNL, Upton, USA, 28.06 - 1.071999

Geneva, Switzerland

July 19, 1999 


\section{Introduction}

Longitudinal coupled bunch instabilities can present a serious limitation to the beam intensity in an accelerator. In the CERN SPS in normal operation the result of these instabilities is a longitudinal emittance blow-up of the proton beam by almost a factor 10 during the acceleration cycle, [1]. It is not harmful for the present fixed target beam operation, total intensity $4.7 \times 10^{13}$, but may become a problem for future roles of the SPS as LHC injector or as a high intensity machine for neutrino experiments.

There are several possible solutions to this problem. They are for example: increased passive damping of resonant impedances, increased Landau damping by applying a high harmonic RF system, active damping using a specially designated feedback system and others. At the moment the most efficient is to use the 4-th harmonic RF system in bunch shortening mode through the cycle. This can stabilize the beam almost up to the top energy $(450 \mathrm{GeV})$, [2]. Nevertheless in all cases identification of the source of instability seems to be an important issue.

For the single bunch in the SPS it was possible to use measurements of the unstable spectrum to determine the sources of microwave instability. For multi-bunch operation, the development of coupled bunch instabilities after transition crossing towards higher energies, is associated with the growth of a broad-band spectrum, see examples in Fig.1.
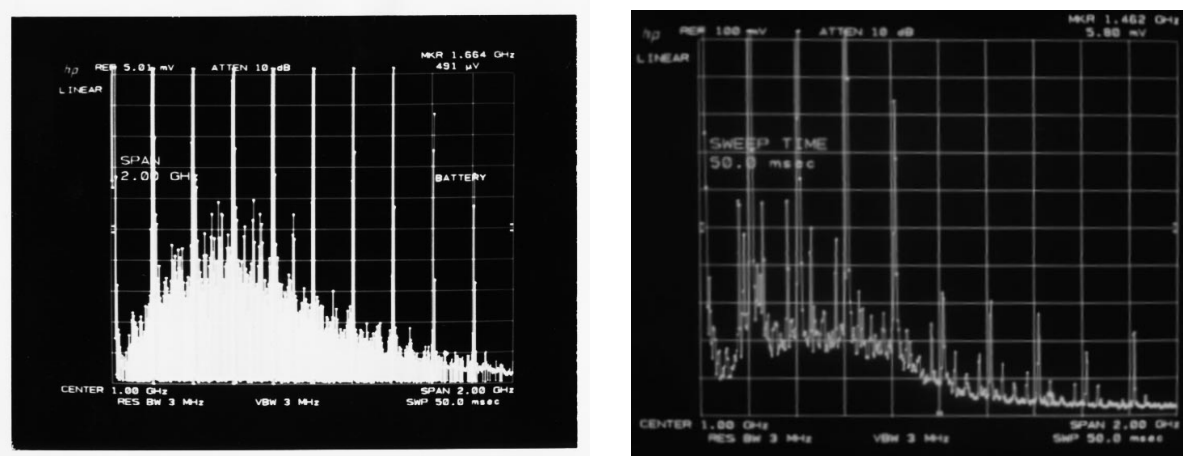

Figure 1: Beam spectrum from 0 to $2 \mathrm{GHz}$ just after transition crossing (left) and at the end of fixed target proton cycle in the CERN SPS (right) for beam intensity $4.2 \times 10^{13}$ (left) and $1.6 \times 10^{13}$ (right). $200 \mathrm{MHz}$ vertical lines are stable beam spectrum.

The spectrum of the unstable bunched beam has components at frequencies

$$
\omega=(n+l M) \omega_{0}+m \omega_{s}
$$

where $\omega_{0}=2 \pi f_{0}$ and $\omega_{s}=2 \pi f_{s}$ are revolution and synchrotron frequencies, $\mathbf{M}$ is the number of bunches in the ring, $n=0,1 \ldots M-1$ is the coupled bunch mode number, describing the phase shift $2 \pi n / M$ between adjacent bunches, $m=0,1 \ldots$ is the multipole number $(\mathrm{m}=1$ - dipole, 2 - quadrupole and so on) and $l=0, \pm 1, \ldots$ Often measurement of $n$ alone is sufficient to guess which HOM in the cavity drives this instability. However sometimes it is not obvious, as in the case of the SPS with its 5 different RF systems installed in the ring and many other cavity-like objects. Then any additional information about the frequency of the guilty impedance would be appreciated. Below we show that under certain conditions the envelope of the longitudinal coupled bunch mode spectrum contains this information. This analysis is then applied to the SPS. 


\section{Review of theory}

\subsection{Main equations}

Let us consider an accelerator with $M$ identical and equally spaced bunches. A general equation describing stability of the system in the presence of coupling impedance $Z(\omega)$ can be obtained from the equations of motion and linearised to give the first order perturbation Vlasov equation, see [3], [4]. This equation can be written in the form:

$$
j_{k}=\sum_{l^{\prime}=-\infty}^{\infty} G_{k k^{\prime}} \frac{Z_{k^{\prime}}}{k^{\prime}} j_{k^{\prime}}
$$

where $k=n+l M, k^{\prime}=n+l^{\prime} M,-\infty<l, l^{\prime}<\infty, Z_{k}=Z\left(k \omega_{0}+\Omega\right)$ and $j_{k}=j\left(k \omega_{0}+\Omega\right)$ is the Fourier transform of the beam current perturbation

$$
j(\theta, t)=e^{i \Omega t} \int_{-\infty}^{\infty} j(\omega) e^{-i \frac{\omega}{\omega_{0}} \theta} \frac{d \omega}{\omega_{0}} .
$$

The elements of matrix $G_{k k^{\prime}}$ are

$$
G_{k k^{\prime}}=A \sum_{m=-\infty}^{\infty} m \omega_{s 0} \int_{0}^{\infty} \frac{d \mathcal{F}}{d r} \frac{I_{m k}(r) I_{m k^{\prime}}^{*}(r)}{\Omega-m \omega_{s}(r)} d r
$$

where

$$
A=-i \frac{J_{A}}{V_{0} h \cos \left(h \theta_{s}\right) S} .
$$

Here $J_{A}$ is the average beam current, $V_{0}$ is the RF voltage amplitude, $h \theta_{s}$ is the synchronous phase, $h$ is the RF harmonic number, $\mathcal{F}(r)$ is the unperturbed distribution function and

$$
I_{m k}(r)=\frac{1}{2 \pi} \int_{-\pi}^{\pi} e^{i k \theta(r, \psi)-i m \psi} d \psi
$$

The normalisation factor $S$ is defined as

$$
S=\omega_{s 0} \int_{0}^{\infty} \frac{\mathcal{F}(r) r d r}{\omega_{s}(r)} .
$$

To describe the system two sets of variables are used: $(\theta, \dot{\theta})$, where $\theta$ is the azimuthal coordinate in the rotating coordinate system, and $(r, \psi)$ - the amplitude and phase of the synchrotron oscillations. For small oscillations with linear synchrotron frequency $\omega_{s 0}$ and maximum oscillation amplitude $r_{\max } \simeq$ $\omega_{0} \tau / 2$, we have $r^{2}=\frac{\dot{\theta}^{2}}{\omega_{s 0}^{2}}+\theta^{2}$. Here $\tau$ is the bunch length in seconds.

Equation (1) can be used for instability threshold calculations in very general cases taking into account the nonlinearity of synchrotron motion (long bunches), an arbitrary RF waveform (double RF system) and so on, see for example [5]. Below we will restrict ourselves to linear synchrotron motion in the single RF system. Then the integral defined in (3) is $I_{m k} \simeq i^{m} J_{m}(k r)$, where $J_{m}(x)$ is the Bessel function of order $m$.

We will consider the excitation of a single multipole $m$ for given frequency $\Omega$ and will discuss this assumption later.

Assuming that $\Omega \ll \omega_{0}$ we neglect the dependence on $\Omega$ in $j_{k}$ and $Z_{k}{ }^{1}$ This allows us to simplify eq.(1) to the following eigenvalue problem, [6]:

$$
\frac{\Omega-m \omega_{s 0}}{m \omega_{s 0}} j_{k}=A \sum_{l^{\prime}} g_{k k^{\prime}}^{m} \frac{Z_{k^{\prime}}}{k^{\prime}} j_{k^{\prime}}
$$

\footnotetext{
${ }^{1}$ We exclude the Robinson instability from consideration.
} 
where

$$
g_{k k^{\prime}}^{m}=\int_{0}^{\infty} \frac{d \mathcal{F}}{d r} J_{m}(k r) J_{m}\left(k^{\prime} r\right) d r .
$$

Eigenvalues of this equation give the frequency shifts of the coherent modes and the eigenfunctions their spectrum. Below we will concentrate on the properties of these eigenfunctions.

\subsection{Analysis of beam spectra}

Let us consider the narrow band impedance with bandwidth $\Delta \omega_{r} \ll M \omega_{0}$. The most simple case for analysis is when the resonant frequency of the impedance $\omega_{r}=2 \pi f_{r}=p_{r} \omega_{0}$ is far away from an integer or half integer multipole of the bunch spacing frequency $M \omega_{0}$. (More general case is considered in Appendix.) Then in the sum in equation (5) we can keep only one term with $l^{\prime}=l_{p}$, where $p=$ $n+l_{p} M \simeq \pm p_{r}$ and (5) becomes

$$
\lambda j_{k}=A g_{k p}^{m} \frac{Z_{p}}{p} j_{p}
$$

where $k=n+l M$ and $-\infty<l<\infty$.

The coherent frequency shift is

$$
\lambda=\frac{\Omega-m \omega_{s 0}}{m \omega_{s 0}}=-i \frac{J_{A}}{V_{0} h \cos \left(h \theta_{s}\right) S} g_{p p}^{m} \frac{Z_{p}}{p} .
$$

For instability one needs resistance. Since $\operatorname{Re} Z$ is always positive and $g_{p p}^{m}$ - negative, $p$ is positive or negative depending on whether we are above or below transition.

As follows from (7) the unstable spectrum for coherent mode $(m, n)$ consists of lines at frequencies $\omega_{k}=2 \pi f_{k}=(n+l M) \omega_{0}+m \omega_{s},-\infty<l<\infty$. Negative frequencies appear on the spectrum analyzer as lower synchrotron sidebands at $(l+1) M \omega_{0}-n \omega_{0}-m \omega_{s}, 0<l<\infty$. The amplitudes of these lines are defined by an eigenfunction which can be written as [6]

$$
j_{p}=1, \quad j_{k}=g_{k p}^{m} / g_{p p}^{m}
$$

where $g_{k p}^{m}$ is given by expression (6). For negative frequencies $j_{-k}=(-1)^{m} j_{k}$ and for odd $m$ lower sidebands will have a phase shift $\pi$, which of course will not be seen on the spectrum analyzer.

In measurements with low frequency resolution similar spectra will be seen due to the resonant impedance at frequencies $(l M+n) \omega_{0}$ and $[(l+1) M-n] \omega_{0}$. However with high enough frequency resolution, the position of the synchrotron sidebands around the revolution lines $n$ and $M-n$ gives information about which $n$ is driven by the impedance: above transition internal sidebands indicate an impedance situated at a higher frequency and external - at a lower, see also [7]. The opposite is true below transition. However the value $l M$ is still unknown.

To proceed further let us consider the binomial family of distribution functions:

$$
\mathcal{F}(r)=\mathcal{F}_{0}\left(1-\frac{r^{2}}{r_{\text {max }}^{2}}\right)^{\mu}
$$

with $\mu \geq 1$. Then function $g_{k p}^{m}$ can be written as

$$
g_{k p}^{m}=-2 \mu \mathcal{F}_{0} \int_{0}^{1}\left(1-x^{2}\right)^{\mu-1} x J_{m}\left(y_{k} x\right) J_{m}\left(y_{r} x\right) d x,
$$

where $y_{k}=k r_{\text {max }} \simeq \pi f_{k} \tau$ and $y_{r}=p_{r} r_{\max } \simeq \pi f_{r} \tau$. In Fig.2 a few examples of beam spectrum envelope are shown for different sets of parameters $m, \mu$ and $f_{r} \tau$.

From these examples it is not obvious to derive a connection between the shape of the unstable beam spectrum envelope and the resonant frequency, although we notice that for some parameters the maximum of the spectrum is close to the resonant frequency. If we now plot the position of the absolute 

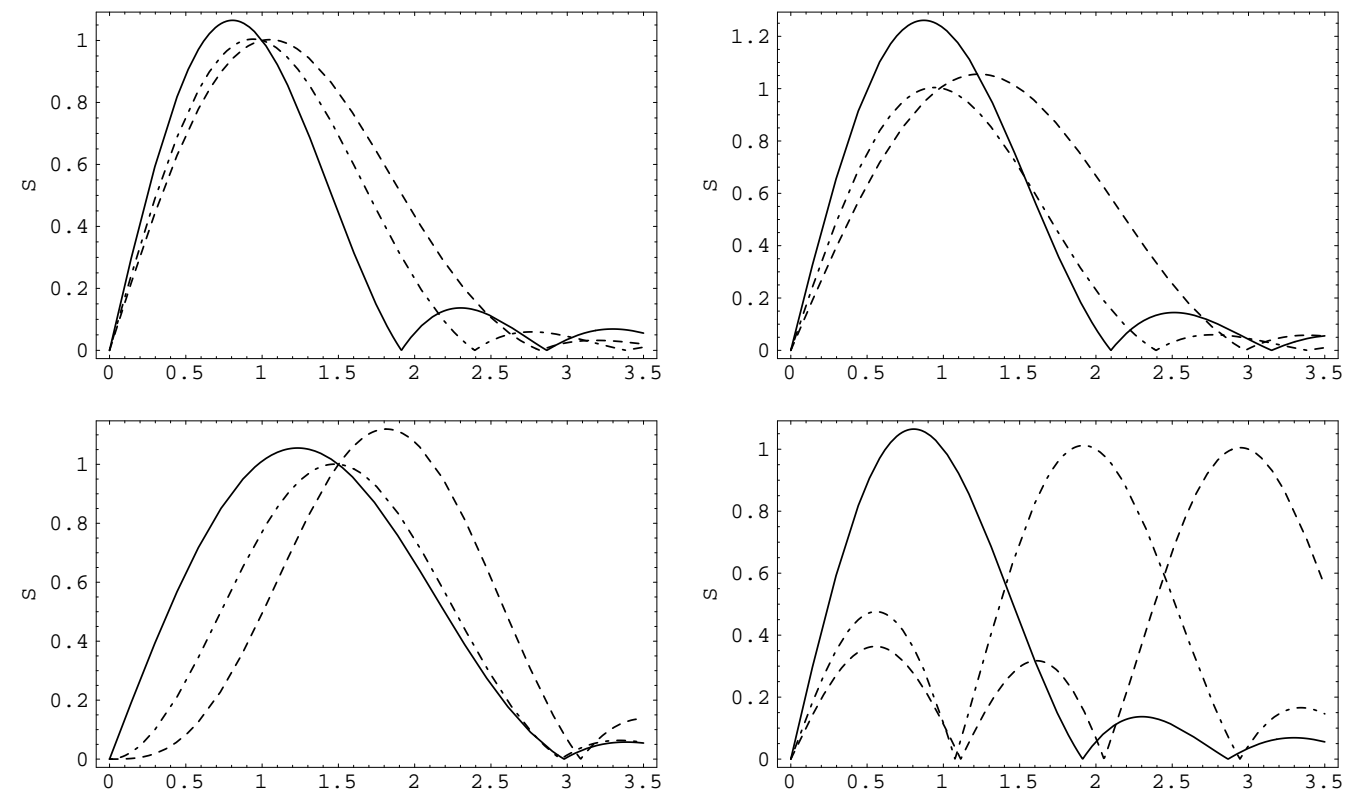

$f_{k} \tau$

$f_{k} \tau$

Figure 2: Beam spectrum envelope for: top, left $-m=1, f_{r} \tau=1$ and $\mu=1.5$ (solid line), $\mu=2$ (dash-dot) and $\mu=2.5$ (dash); top, right $-m=1, \mu=2$ and and $f_{r} \tau=0.5$ (solid), $f_{r} \tau=1$ (dash-dot) and $f_{r} \tau=1.5$ (dash); bottom, left $-f_{r} \tau=1.5, \mu=2$ and $m=1$ (solid), $m=2$ (dash-dot) and $m=3$ (dash); bottom, right - $m=1, \mu=1$ and $f_{r} \tau=1$ (solid), $f_{r} \tau=2$ (dash-dot) and $f_{r} \tau=3$ (dash).
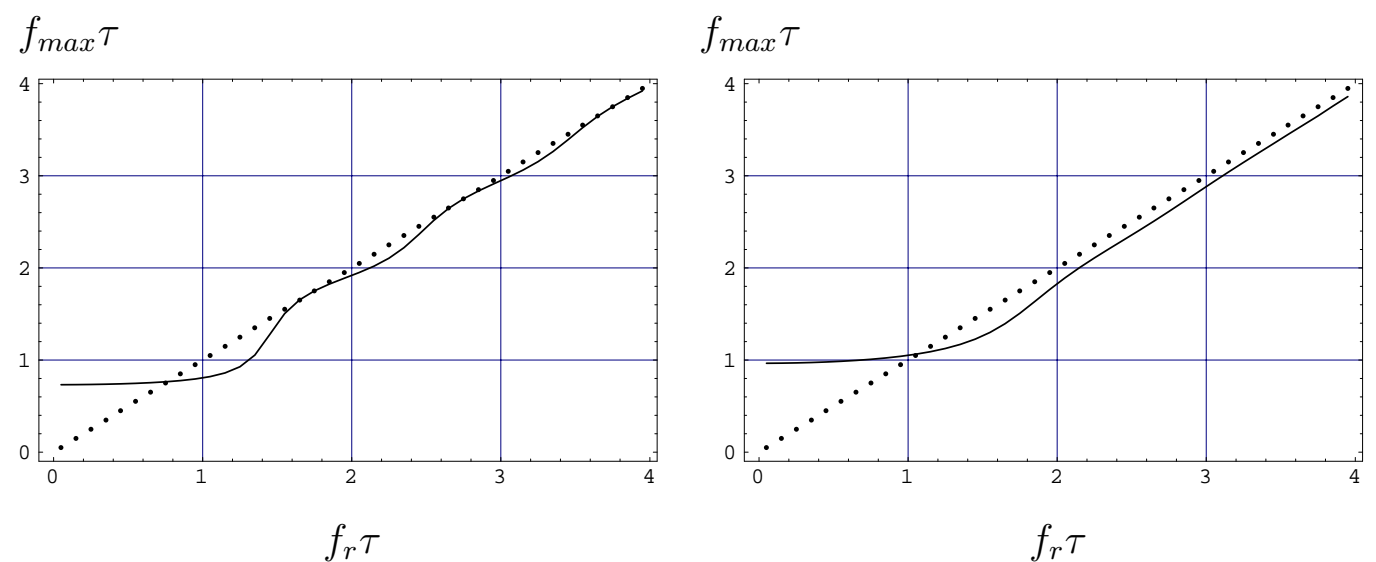

Figure 3: Position of the maximum in the beam spectrum envelope as a function of $f_{r} \tau$ for dipole mode $m=1$ and two different distribution functions with $\mu=1$ (left) and $\mu=3$ (right).

maximum $f_{\text {max }} \tau$ as a function of the resonant frequency $f_{r} \tau$ (both normalised to bunch length) we get dependencies as shown in Fig. 3 for the dipole mode $m=1$ and the distribution function with $\mu=1$ (left) and $\mu=3$ (right). One can clearly see two different regimes: one, when $f_{\max } \tau \simeq$ const and another, when $f_{\max } \simeq f_{r}$. The transition between them for $m=1$ occurs around $f_{r} \tau \sim 1$. The const is in fact a function of $\mu$ and $m$. Similar behaviour for the maximum of the spectrum envelope can be observed for higher multipoles $m$. Examples for $m=2,3$ and $\mu=1$ are presented in Fig.4.

The existence of two different regimes can formally be understood from the behaviour of the Bessel 
$f_{\text {max }} \tau$

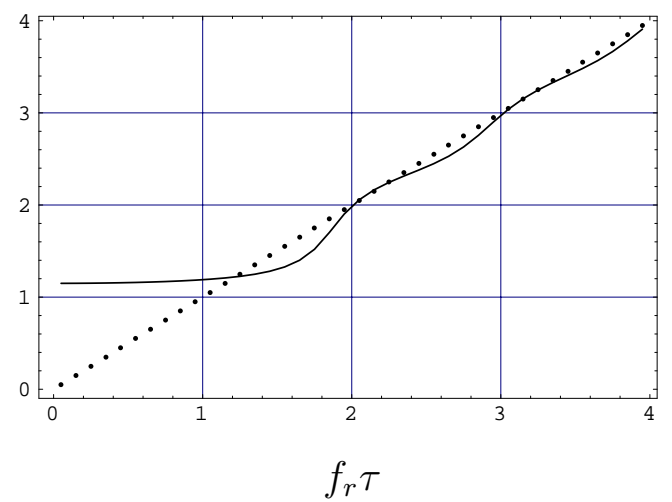

$f_{\max } \tau$

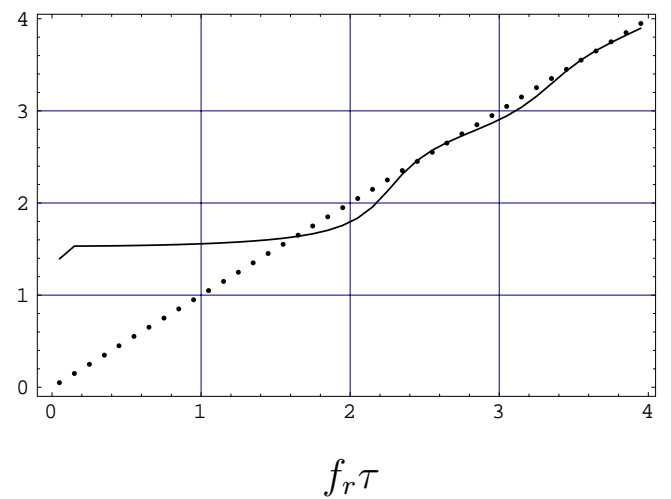

Figure 4: Position of the maximum in the beam spectrum envelope as a function of $f_{r} \tau$ for $\mu=1$ and modes $m=2$ (left) and $m=3$ (right).

functions for small and large arguments. Using the first term in the expansion of $J_{m}\left(y_{r} x\right)$ for $y_{r}<1$ we see that

$$
j_{k} \propto \int_{0}^{1} x\left(1-x^{2}\right)^{\mu-1} x^{m} J_{m}\left(y_{k} x\right) d x
$$

and the position of the maximum does not depend on $y_{r}$. For $\mu=1$ the beam spectrum has amplitudes

$$
j_{k} \propto \frac{J_{m+1}\left(y_{k}\right)}{y_{k}}
$$

The asymptotic behaviour of the Bessel functions for large arguments $x \gg 1$

$$
J_{m}(x) \simeq\left(\frac{2}{\pi x}\right)^{1 / 2} \cos \left(x-\frac{m \pi}{2}-\frac{\pi}{4}\right)
$$

explains why starting from some value of $f_{r} \tau$ the spectrum peaks at $f_{\text {max }} \sim f_{r}$. Indeed, the function under the integral in (11) is zero at $x=0$ and the error using this asymptotic at small $x$ can be shown to be of the order $\ln \left(y_{r}\right) /\left(y_{r}\right)$. Then for $y_{r}, y_{k}>1$

$$
j_{k} \sim \frac{1}{y_{k}^{1 / 2}} \int_{0}^{1}\left(1-x^{2}\right)^{\mu-1} \cos \left[\left(y_{k}-y_{r}\right) x\right] d x .
$$

For $\mu=1$ this gives

$$
j_{k} \sim \frac{\sin \left(y_{k}-y_{r}\right)}{y_{k}^{1 / 2}\left(y_{k}-y_{r}\right)} .
$$

These results have a clear physical meaning as well. If the wavelength of the wake field is longer than the bunch length, the spectrum of the perturbation does not contain any information about it. For higher frequencies or smaller wavelength the bunch length is already sufficiently long to "resolve" the frequency of the perturbation. The most efficiently excited is the perturbation with a maximum of the spectrum closest in frequency to the driving impedance.

From this analysis we can already conclude that for a fixed bunch length the resonant frequency of the impedance driving the instability is either below or very close (and slightly above) the maximum of the bunch spectrum envelope. However if one also takes into account values of $f_{r} \tau$ at which the given mode $m$ is most efficiently excited then, as we will see below, for all modes, except dipole, the maximum of the spectrum is close to the resonant frequency. 
To understand under which conditions a given spectrum will be excited, one should consider simultaneously the thresholds or growth rates for different modes $m .{ }^{2}$ Growth rates (8) for the distribution function (10) can be presented in the form:

$$
\frac{\operatorname{Im} \Omega}{\omega_{s}}=\frac{4}{\pi^{2}} \frac{J_{A} \operatorname{Re} Z}{h V_{0} \cos \left(h \theta_{s}\right)} \frac{F_{m}^{*}}{f_{0} \tau}, \quad F_{m}^{*}=\frac{m \mu(\mu+1)}{f_{r} \tau} \int_{0}^{1} x\left(1-x^{2}\right)^{\mu-1} J_{m}^{2}\left(y_{r} x\right) d x .
$$

In Fig.5 the dependence of the formfactor $F_{m}^{*}$ on $f_{r} \tau=y_{r} / \pi$ for modes $m=1,2,3,4$ is shown for distribution functions with $\mu=1$ (left) and $\mu=2$ (right).
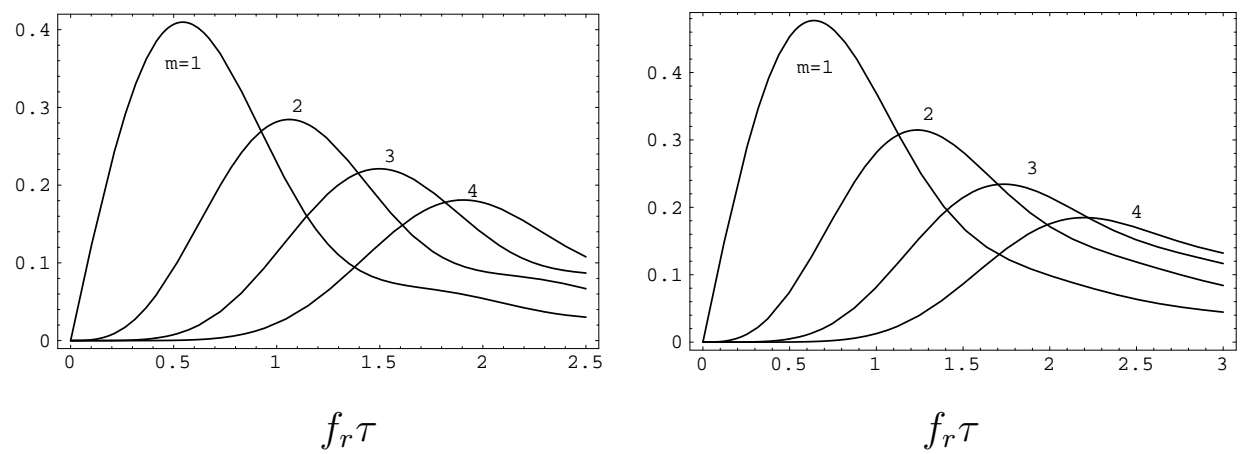

Figure 5: Formfactor $F_{m}^{*}$ from eq.(16) for modes $m=1,2,3,4$ as a function of $f_{r} \tau$ for distributions with $\mu=1$ (left) and $\mu=2$ (right).

From Fig. 5 one can notice that for $\mu=1$ mode $m=1$ is dominant up to $f_{r} \tau \simeq 0.9, m=2$ from this value up to $1.4, m=3$ from 1.4 to 1.85 and so on... At low values of $f_{r} \tau$ higher modes are strongly supressed in comparison with the dipole mode. Then we see that in Fig.4 in the first regime ( $f_{\max } \tau \simeq$ const) only the part of the curve which is close to the diagonal has sense.

Above we considered independent excitation of the different modes $\mathrm{m}$. This assumption is valid for bunches with small synchrotron frequency spread and resonant frequency which is not too high [5]. If one of these conditions is not satisfied, then many multipoles can be excited simultaneously. Then it is possible, [5], [8], to sum up terms with different $m$ in equation (1). This situation describes the so called microwave instability with a spectrum having a maximum at the frequency of the resonant impedance.

\subsection{Coupled bunch instabilities in the CERN SPS}

Calculations show [9] that after transition crossing during the fixed target cycle in the SPS the threshold for coupled bunch instabilities is continuously decreasing. It also has a frequency dependence so that the lowest threshold is for $f_{r} \sim 0.4 / \tau$. As a result, different HOM can be successively excited during the cycle due to a bunch length change.

One other example of the bunch spectrum during the development of a coupled bunch instability towards the end of the cycle in the CERN SPS is shown in Fig.6. This beam had a total intensity 10 times lower than in the measurements presented in Fig.1 (left). As one can notice, at this intensity the signals are much clearer.

From the measured value of $n f_{0} \sim 113 \mathrm{MHz}$ a few candidates were possible. These measurements were done with at low intensity. At the end of the cycle the bunch length was (1.5 - 2) ns, significantly shorter than in the measurements presented in Fig.1 (left) for the high intensity beam (2.5 - $3 \mathrm{~ns}$ ). However both spectra have a maximum around $700 \mathrm{MHz}$. In both cases parameter $f_{\text {max }} \tau$ is more than 1. From this and also the fact that the maximum is the same, we expect the resonant frequency to be close to the maximum of the spectrum. This can be a HOM in the travelling wave $200 \mathrm{MHz}$ RF system with resonant frequency $912 \mathrm{MHz}$.

\footnotetext{
${ }^{2}$ In this analysis bunch length is assumed constant.
} 

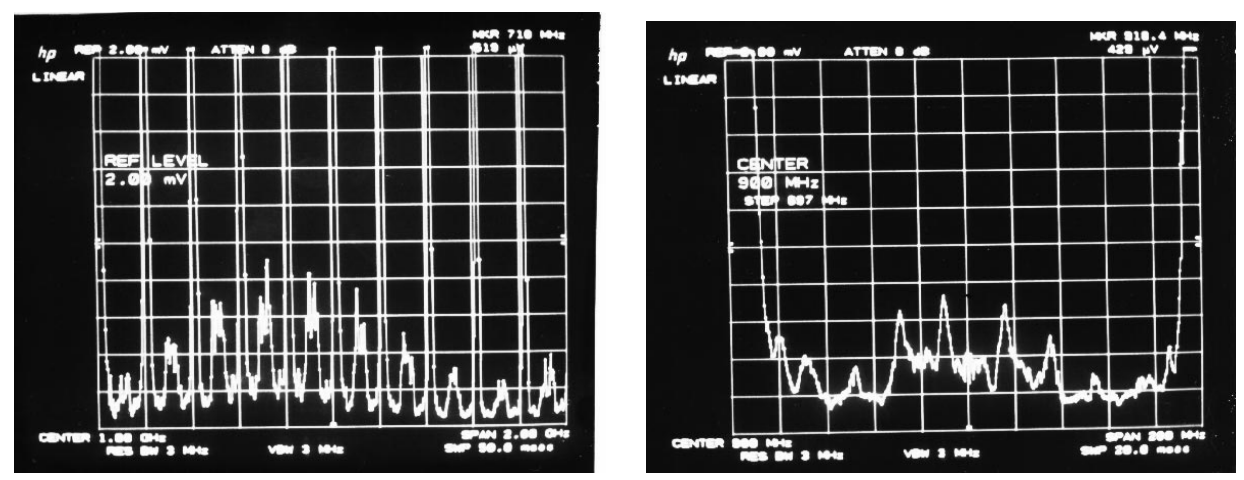

Figure 6: Beam spectrum from 0 to $2 \mathrm{GHz}$ (left) and from $0.8 \mathrm{GHz}$ to $1 \mathrm{GHz}$ (right) at the end of the fixed target proton cycle in the CERN SPS for low beam intensity $4 \times 10^{12}$.

In Fig. 1 (right) we presented the signal obtained with quite long bunches, during the development of instability due to the fundamental frequency of the $352 \mathrm{MHz}$ superconducting RF system, installed in the SPS for lepton acceleration, when non-optimal passive damping was accidentally used. In this case the spectrum had a maximum close to the resonant frequency.

\section{Conclusions}

As a conclusion we summarise which additional information about the resonant frequency of the guilty impedance can be obtained from the measured beam spectrum:

- The measured bunch profile and the stable beam spectrum can give some idea about the distribution function $(\mu)$

- Measurements of the bunch length $\tau$ and $f_{\max }$ from the unstable bunch spectrum give $f_{\max } \tau$

- Now there are two possibilities:

- parameter $f_{\max } \tau<1$; then one only can say that $f_{r} \tau \leq 1.2$

- parameter $f_{\text {max }} \tau>1$, then the most probable $f_{r} \sim f_{\text {max }}$. The uncertainty can be estimated, for example, from the vertical displacement of the curves from the diagonal and in the region with efficient excitation (see Fig.5) does not exceed $\pm 0.2 / \tau$. The smaller the bunch length - the larger the uncertainty.

\section{Acknowledgements}

I am grateful to T.Linnecar and T.Bohl for stimulating discussions and support.

\section{Appendix}

Now let us consider the possibility that the resonant frequency of the narrow-band impedance is close to an integer or half integer multipole of the bunch spacing frequency $M \omega_{0}$. Then we should keep two terms in the sum in equation (5) and it becomes

$$
\lambda j_{k}=A g_{k p_{1}}^{m} \frac{Z_{p_{1}}}{p_{1}} j_{p_{1}}+A g_{k p_{2}}^{m} \frac{Z_{p_{2}}}{p_{2}} j_{p_{2}},
$$

where $p_{1}=p=n+l_{1} M \simeq \pm p_{r}$ and $p_{2}=n+l_{2} M$. Here $p_{1} p_{2}<0$ and for values of $l_{2}$ we have 2 possibilities, depending on where the resonant impedance is situated: $l_{2}=-l_{1}$ or $l_{2}=-\left(l_{1}+1\right)$. 
Taking for $k$ values $p_{1}$ and $p_{2}$ we can find eigenvalues for this situation. For the narrow band impedance assumed above $\Delta \omega_{r} \ll M \omega_{0} \ll 1 / \tau$. In this case we can write

$$
\begin{aligned}
& g_{p_{1} p_{1}}^{m} \simeq \quad g_{p_{2} p_{2}}^{m} \quad=g_{p}^{m} \\
& g_{p_{1} p_{2}}^{m}=g_{p_{2} p_{1}}^{m} \simeq(-1)^{m} g_{p}^{m} \\
& g_{k p_{1}}^{m} \simeq(-1)^{m} g_{k p_{2}}^{m} .
\end{aligned}
$$

Then for the growing mode, the eigenvalue is

$$
\lambda \simeq A g_{p}^{m}\left(\frac{Z_{p_{1}}}{p_{1}}+\frac{Z_{p_{2}}}{p_{2}}\right) .
$$

There is no instability if $p_{1}=-p_{2}$, which can happen [4] when the $\omega_{r}$ is equal to an integer or half integer (for even $M$ ) multipole of the bunch spacing frequency $M \omega_{0}$.

For the beam spectrum we finally have an expression similar to (1):

$$
j_{k}=A\left[\frac{Z_{p_{1}}}{p_{1}} g_{k p_{1}}^{m}+(-1)^{m} \frac{Z_{p_{2}}}{p_{2}} g_{k p_{2}}^{m}\right] / \lambda \simeq g_{k p}^{m} / g_{p}^{m} .
$$

\section{References}

[1] T.Bohl, T.Linnecar, E.Shaposhnikova, Emittance control by the modification of the voltage programme, CERN SL-MD Note 246, 1997.

[2] T.Bohl, T.Linnecar, E.Shaposhnikova, J.Tückmantel, Study of different operating modes of the 4th RF harmonic Landau damping system in the CERN SPS, proceed. EPAC'98, 1998.

[3] A.N.Lebedev, Proc. VI Int. Conf. on H.E. Accel., CEAL-2000, p.284, 1967; Atomnaya Energia, v.25, N2, p.100, 1968.

[4] F.J.Sacherer, A longitudinal stability criterion for bunched beams, IEEE Trans. Nucl. Sci. NS-20, p.825, 1973.

[5] V.I.Balbekov, S.V.Ivanov, Longitudinal beam instabilities in the proton synchrotrons, Proc. XIII Int. Conf. H.E. Accel., Novosibirsk, v.2, p.124, 1987, in Russian.

[6] J.L.Laclare, Bunched beam coherent instabilities, CERN Acc. School 1985, CERN 87-03, p.264, 1987.

[7] F.Sacherer, F.Pedersen, Theory and performance of the longitudinal active damping system for the CERN PS Bosster, NS-24, N.3, p.1396, 1977.

[8] J.M.Wang, C.Pelegrini, On the condition for a single bunch high frequency fast blow-up, Proc. XI Int. Conf. on H.E. Accel., Geneva, p.554, 1980.

[9] E.Shaposhnikova, Longitudinal instabilities in the SPS, Proc. of the workshop on LEP-SPS performance, Chamonix IX, Chamonix, p.69, 1999. 